Supporting Information for:

\title{
Reaction of Copper(II) Chloroacetate with Pyrazole. Synthesis of a One- Dimensional Coordination Polymer and Unexpected Dehydrochlorination
} Reaction.

Silvia Carlotto, ${ }^{a}$ Maurizio Casarin, ${ }^{a^{*}}$ Arianna Lanza, ${ }^{b^{*}}$ Fabrizio Nestola, ${ }^{c}$ Luciano Pandolfo, ${ }^{a^{*}}$ Claudio Pettinari, ${ }^{d}$ Rebecca Scatena. ${ }^{\text {a }}$

a'Dipartimento di Scienze Chimiche, Università di Padova, Via Marzolo, 1, I-35131 Padova, Italy.

${ }^{b}$ Department für Chemie und Biochemie, Universität Bern, Freiestrasse, 3, CH-3012 Bern, Switzerland.

'Dipartimento di Geoscienze, University of Padova, Via Gradenigo, 6, I-35131 Padova, Italy.

${ }^{\text {d}}$ Scuola di Farmacia, University of Camerino, Via S. Agostino, 1, I-62032 Camerino (MC), Italy. 


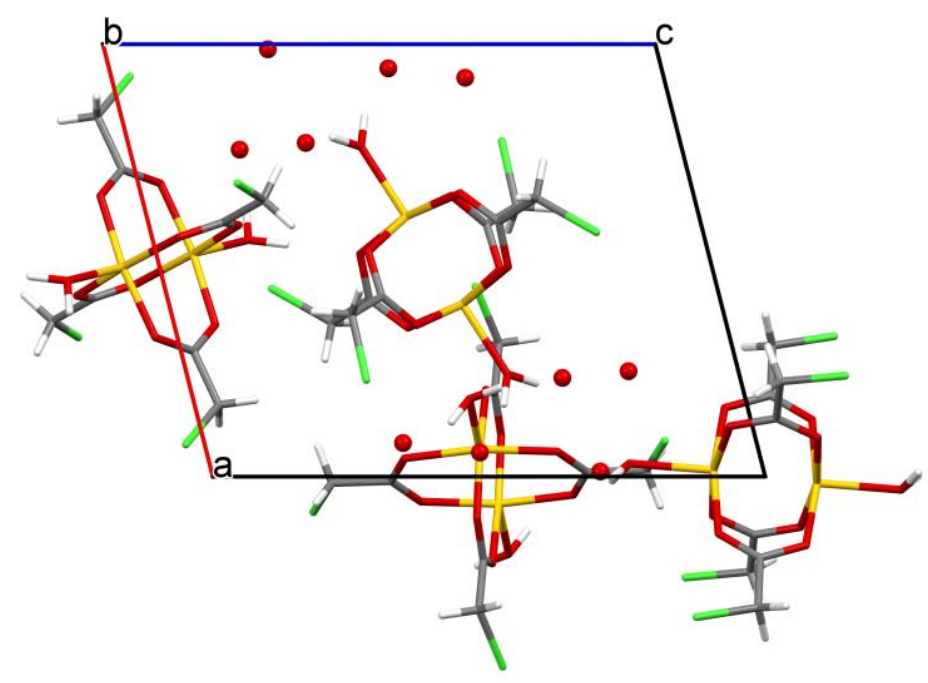

Figure S1 - Capped-stick representation of the crystal cell of hydrated copper(II) chloroacetate $\left\{\left[\mathrm{Cu}(\mathrm{Clac})_{2}\left(\mathrm{H}_{2} \mathrm{O}\right)\right]_{2}\right\}_{4} \cdot 10 \mathrm{H}_{2} \mathrm{O}, 1 \mathrm{~d}$. Crystallization water molecules ( $\mathrm{H}$ atoms were not located in the Fourierdifference map) are evidenced in ball-and-stick representation.

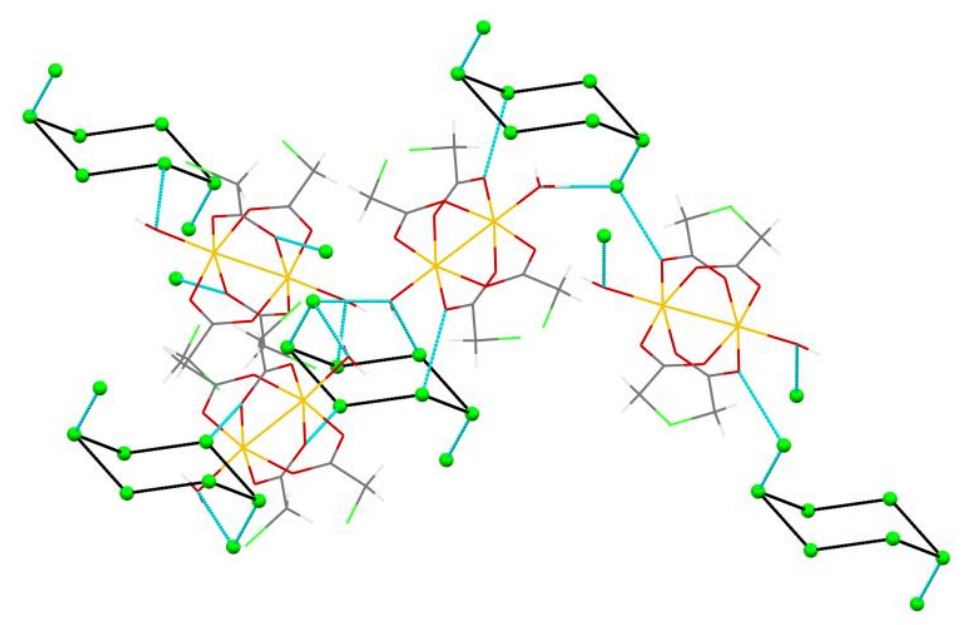

Figure S2 - H-bonds (light-blue dotted lines) connecting six-membered cycles of crystallization water molecules (water molecules as green dots, $\mathrm{H}$-bonds as black lines) in compound $\mathbf{1 d}$.
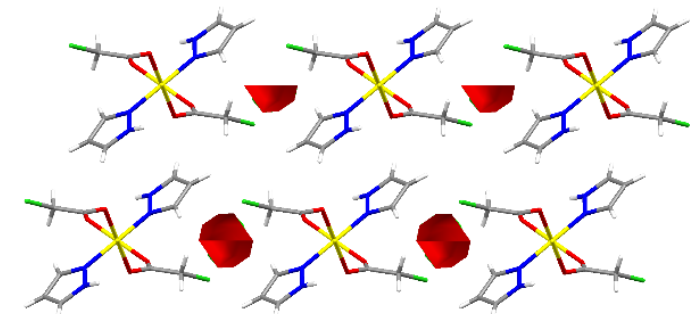

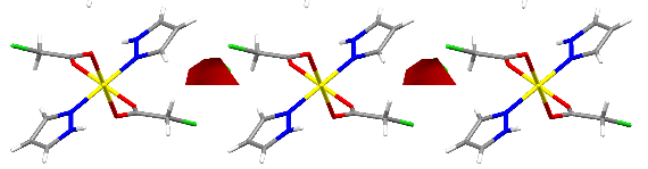

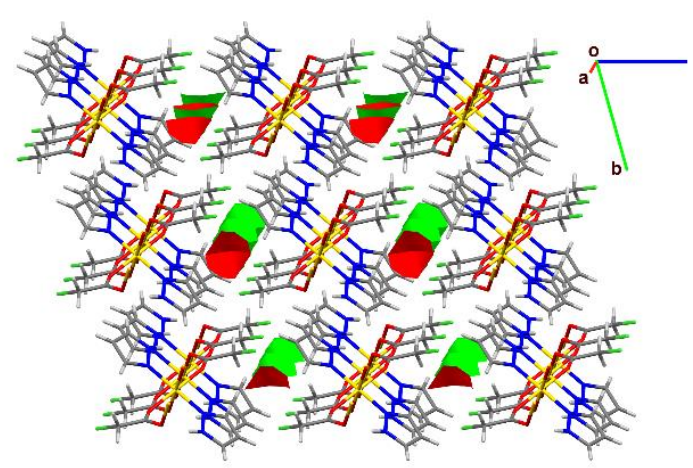

Figure $\mathbf{3} \mathbf{3}$ - Capped stick representations of the crystal packing of $\mathbf{2 m}$ (left view down the crystallographic $a$ axis) evidencing the presence of small pores accounting for about $2 \%$ of free volume. 


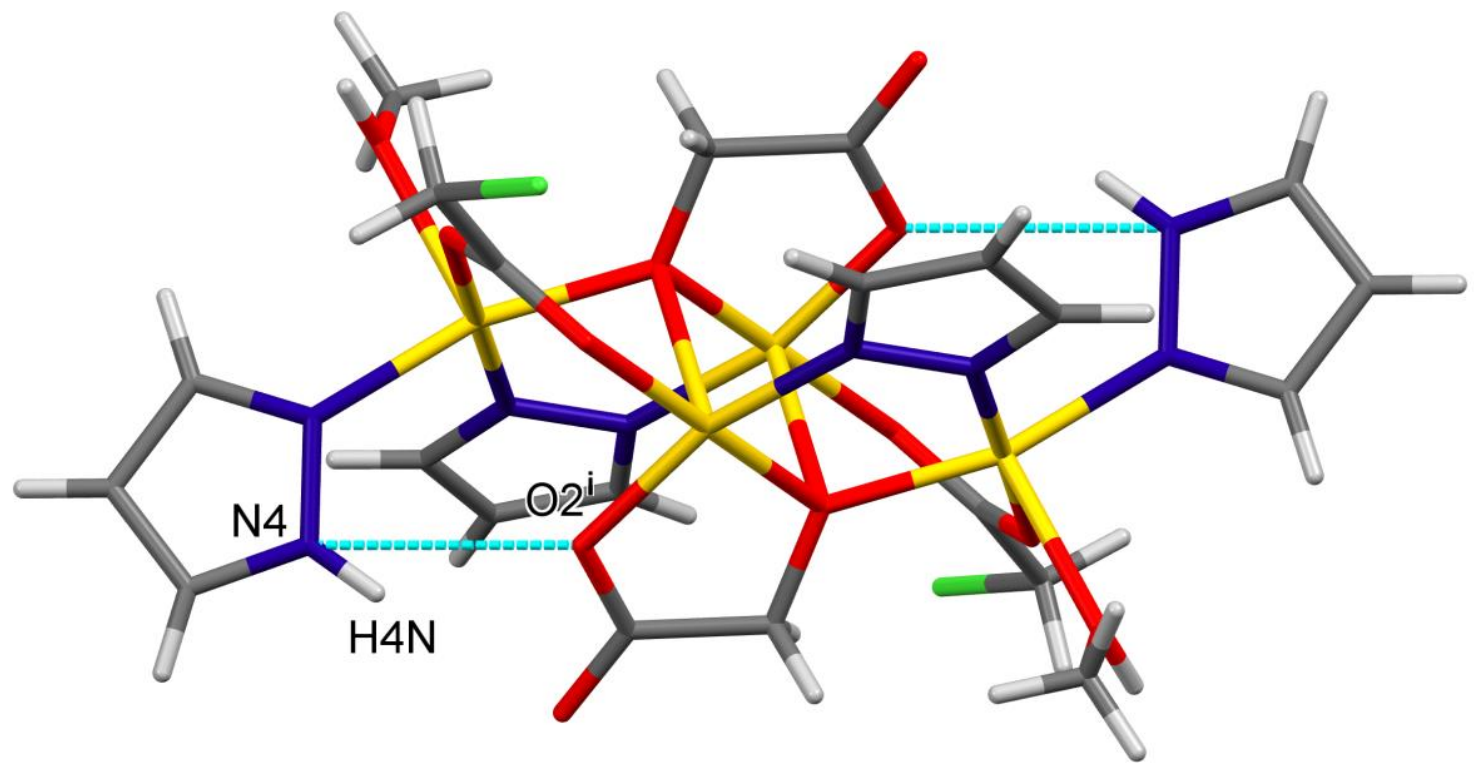

Figure $\mathbf{S 4}$ - View of the tetranuclear assembly of $\mathbf{3 t}$ with intramolecular $\mathrm{H}$-bonds (light blue dashed lines) likely reinforcing the tetranuclear structure.

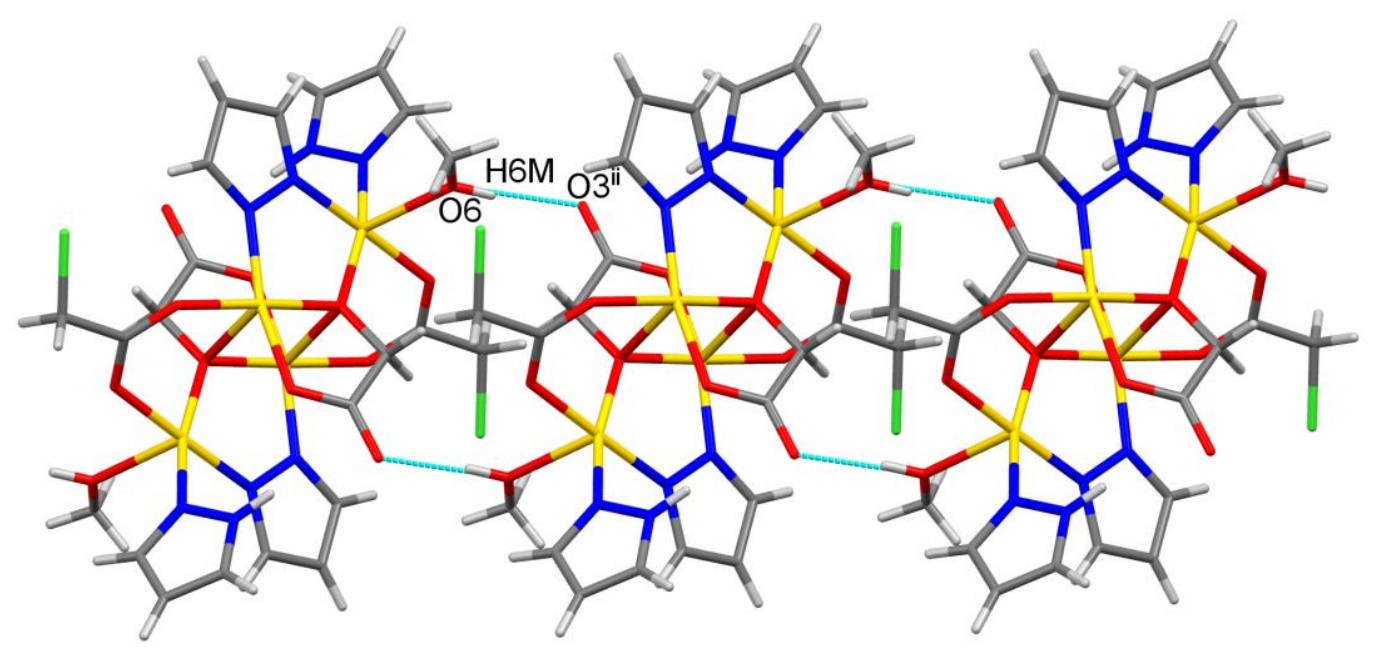

Figure S5 - View of the supramolecular 1D assembly of 3t obtained through intermolecular H-bonds (light blue dashed lines). 
(a)

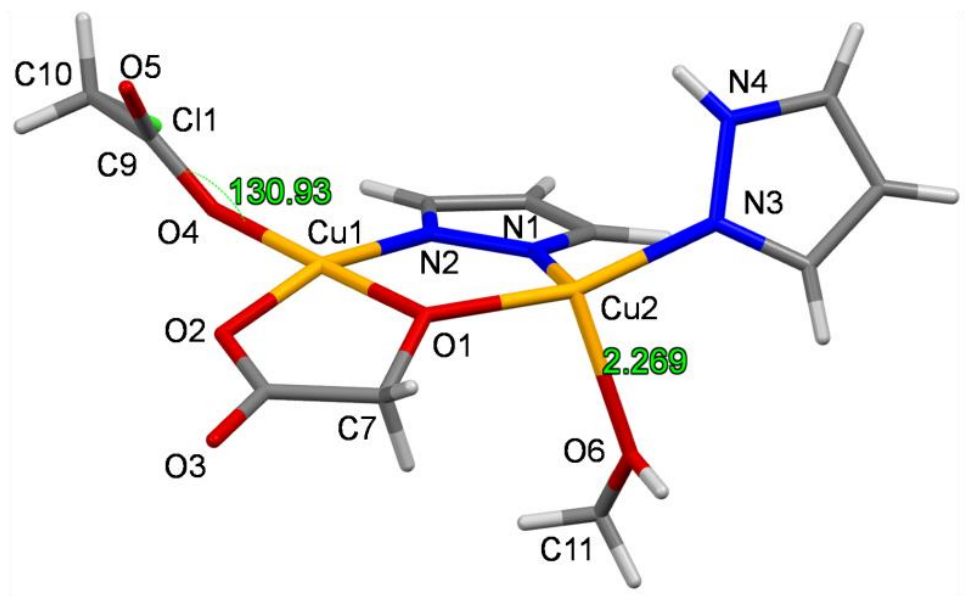

(b)

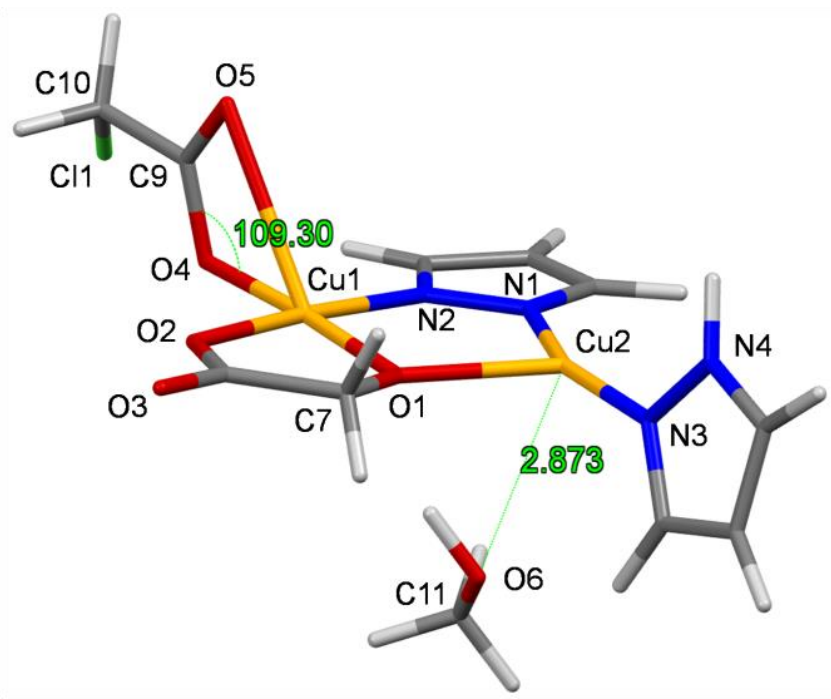

(c)

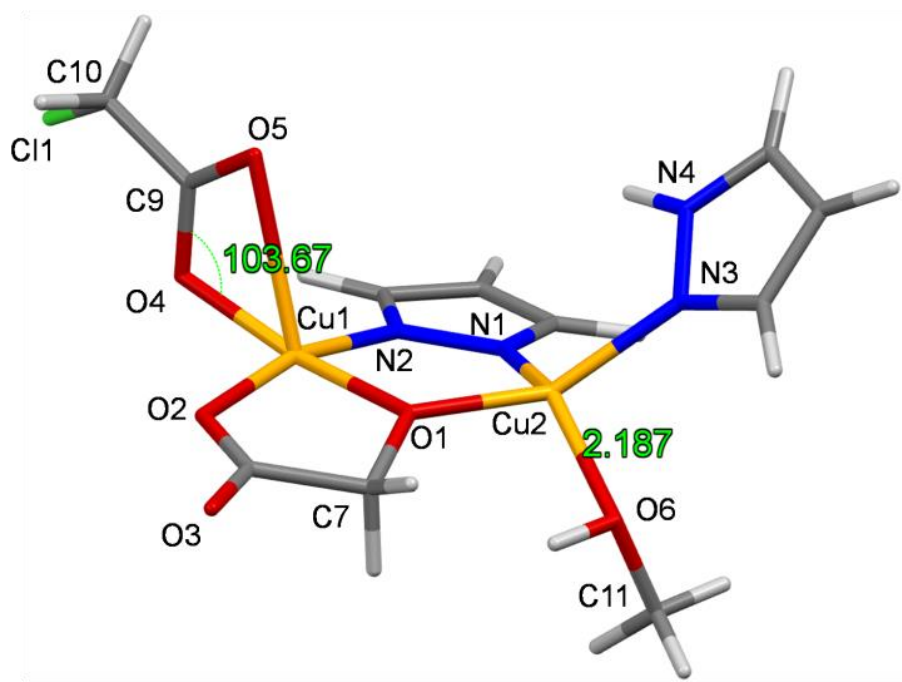

Figure S6 - Licorice representation of the asymmetric unit of $\mathbf{3 t}$ without the inclusion of solvent effects; (a) experimental coordinates, (b) coordinates optimized by assuming an antiferromagnetic coupling $(S=0)$, (c) coordinates optimized by assuming a ferromagnetic coupling $(S=1)$. Bond angles are in deg, while bond distances are in $\AA$. 
Table S1 - Optimized Cartesian coordinates of the asymmetric unit of 3t without the inclusion of solvent effects and by assuming either an antiferromagnetic $(S=0)$ or a ferromagnetic $(S=1)$ coupling.

\begin{tabular}{|c|c|c|c|c|c|c|c|}
\hline \multicolumn{4}{|c|}{$S=0$} & \multicolumn{4}{|c|}{$S=1$} \\
\hline $\mathrm{C}$ & 4.325320 & 2.521416 & 4.314075 & $\mathrm{C}$ & 1.874950 & 2.431715 & 5.875527 \\
\hline C & 5.549294 & 2.889075 & 4.852801 & $\mathrm{C}$ & 2.988534 & 2.228660 & 5.069766 \\
\hline $\mathrm{C}$ & 5.251384 & 3.824705 & 5.858812 & $\mathrm{C}$ & 3.891770 & 3.243948 & 5.426470 \\
\hline $\mathrm{N}$ & 3.921096 & 4.022803 & 5.939663 & $\mathrm{~N}$ & 3.371875 & 4.022771 & 6.393107 \\
\hline $\mathrm{N}$ & 3.374489 & 3.216772 & 4.986286 & $\mathrm{~N}$ & 2.141363 & 3.509783 & 6.648908 \\
\hline $\mathrm{Cu}$ & 2.993692 & 5.115135 & 7.193622 & $\mathrm{Cu}$ & 3.883344 & 5.774373 & 7.226764 \\
\hline $\mathrm{N}$ & 1.979368 & 5.610097 & 8.725880 & $\mathrm{~N}$ & 2.761703 & 5.787125 & 8.848948 \\
\hline $\mathrm{N}$ & 1.740655 & 6.908747 & 9.054493 & $\mathrm{~N}$ & 2.111633 & 6.943666 & 9.183040 \\
\hline $\mathrm{C}$ & 1.118947 & 6.957528 & 10.253321 & $\mathrm{C}$ & 1.333376 & 6.701908 & 10.256669 \\
\hline $\mathrm{C}$ & 0.953792 & 5.654880 & 10.731675 & $\mathrm{C}$ & 1.468786 & 5.362618 & 10.642727 \\
\hline $\mathrm{C}$ & 1.507577 & 4.842264 & 9.739999 & $\mathrm{C}$ & 2.376776 & 4.823518 & 9.727665 \\
\hline $\mathrm{Cu}$ & 2.214251 & 8.363564 & 7.873766 & $\mathrm{Cu}$ & 2.362106 & 8.594691 & 8.133718 \\
\hline $\mathrm{O}$ & 2.790789 & 9.702379 & 6.714939 & 0 & 3.059189 & 10.217214 & 7.271583 \\
\hline C & 3.408666 & 9.279897 & 5.620917 & $\mathrm{C}$ & 4.073862 & 10.045559 & 6.502492 \\
\hline $\mathrm{C}$ & 3.649433 & 7.765500 & 5.566826 & $\mathrm{C}$ & 4.699805 & 8.592822 & 6.538841 \\
\hline $\mathrm{O}$ & 3.202910 & 7.166218 & 6.787039 & 0 & 3.746857 & 7.686724 & 7.014101 \\
\hline $\mathrm{O}$ & 1.333714 & 9.632454 & 8.985068 & 0 & 0.793104 & 9.494852 & 8.909596 \\
\hline C & 0.136955 & 9.868205 & 8.508715 & $\mathrm{C}$ & -0.150442 & 9.260208 & 8.051138 \\
\hline C & -0.649694 & 10.956412 & 9.250591 & $\mathrm{C}$ & -1.522979 & 9.885764 & 8.310881 \\
\hline $\mathrm{Cl}$ & -0.440346 & 10.967420 & 11.056419 & $\mathrm{Cl}$ & -1.930127 & 10.217387 & 10.053591 \\
\hline $\mathrm{O}$ & 5.503648 & 6.196430 & 8.079938 & 0 & 5.923472 & 5.695722 & 6.442215 \\
\hline C & 5.706911 & 6.463929 & 9.479220 & $\mathrm{C}$ & 6.984583 & 5.306198 & 7.359109 \\
\hline 0 & 3.756360 & 10.047605 & 4.735454 & 0 & 4.648650 & 10.860902 & 5.776996 \\
\hline 0 & -0.371987 & 9.334971 & 7.520013 & 0 & -0.019497 & 8.601006 & 7.003041 \\
\hline $\mathrm{H}$ & 1.592585 & 3.760625 & 9.691919 & $\mathrm{H}$ & 2.798508 & 3.822581 & 9.670685 \\
\hline $\mathrm{H}$ & 0.492663 & 5.348595 & 11.664506 & $\mathrm{H}$ & 0.986868 & 4.859640 & 11.474806 \\
\hline $\mathrm{H}$ & 0.841726 & 7.913344 & 10.686181 & $\mathrm{H}$ & 0.726658 & 7.501500 & 10.671776 \\
\hline $\mathrm{H}$ & 5.899322 & 4.378399 & 6.533960 & $\mathrm{H}$ & 4.881047 & 3.463230 & 5.037427 \\
\hline $\mathrm{H}$ & 4.061376 & 1.833562 & 3.517661 & $\mathrm{H}$ & 0.931377 & 1.901539 & 5.949986 \\
\hline $\mathrm{H}$ & 4.769925 & 6.776718 & 7.762446 & $\mathrm{H}$ & 6.099043 & 6.613440 & 6.148712 \\
\hline $\mathrm{H}$ & 2.367952 & 3.201857 & 4.858561 & $\mathrm{H}$ & 1.570793 & 3.957828 & 7.366613 \\
\hline $\mathrm{H}$ & 6.527743 & 2.529626 & 4.556320 & $\mathrm{H}$ & 3.123423 & 1.453486 & 4.323870 \\
\hline $\mathrm{H}$ & 3.096936 & 7.355193 & 4.706110 & $\mathrm{H}$ & 5.045053 & 8.343637 & 5.518641 \\
\hline $\mathrm{H}$ & 4.725892 & 7.588156 & 5.415997 & $\mathrm{H}$ & 5.568432 & 8.700550 & 7.218753 \\
\hline $\mathrm{H}$ & 5.996190 & 7.512722 & 9.651439 & $\mathrm{H}$ & 7.004371 & 5.958949 & 8.242423 \\
\hline $\mathrm{H}$ & 6.524859 & 5.816891 & 9.818011 & $\mathrm{H}$ & 7.954037 & 5.332976 & 6.844013 \\
\hline $\mathrm{H}$ & 4.806694 & 6.236938 & 10.072576 & $\mathrm{H}$ & 6.764251 & 4.279664 & 7.668634 \\
\hline $\mathrm{H}$ & -1.713419 & 10.812421 & 9.044708 & $\mathrm{H}$ & -2.294295 & 9.217236 & 7.918141 \\
\hline $\mathrm{H}$ & -0.321064 & 11.937869 & 8.887568 & $\mathrm{H}$ & -1.571937 & 10.847793 & 7.786163 \\
\hline
\end{tabular}


(a)
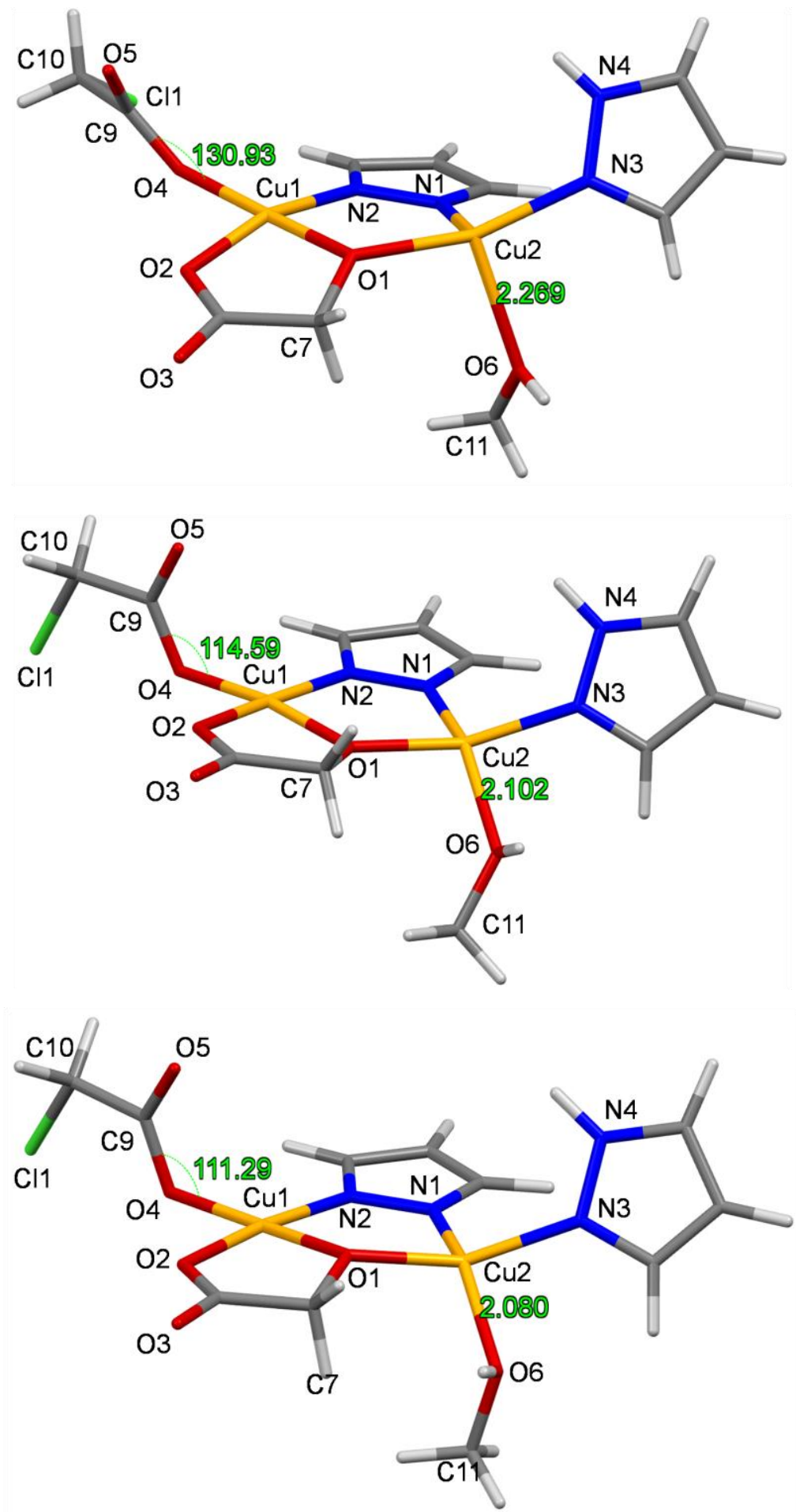

Figure S7 - Licorice representation of the asymmetric unit of $\mathbf{3 t}$ with the inclusion of solvent (methanol) effects; (a) experimental coordinates, (b) coordinates optimized by assuming an antiferromagnetic coupling $(S=0)$, (c) coordinates optimized by assuming a ferromagnetic coupling $(S=1)$. Bond angles are in deg, while bond distances are in $\AA$. 
Table S2 - Optimized Cartesian coordinates of the asymmetric unit of 3t with the inclusion of solvent effects and by assuming either an antiferromagnetic $(S=0)$ or a ferromagnetic $(S=1)$ coupling.

\begin{tabular}{|c|c|c|c|c|c|c|c|}
\hline \multicolumn{4}{|c|}{$S=0$} & \multicolumn{4}{|c|}{$S=1$} \\
\hline C & 3.082972 & 1.939063 & 5.325849 & $\mathrm{C}$ & 2.897049 & 1.946682 & 5.531172 \\
\hline C & 4.288691 & 1.592939 & 5.926176 & $\mathrm{C}$ & 4.205533 & 1.652928 & 5.900341 \\
\hline$C$ & 4.732861 & 2.762429 & 6.562947 & $\mathrm{C}$ & 4.714647 & 2.843192 & 6.440650 \\
\hline $\mathrm{N}$ & 3.859957 & 3.771651 & 6.363796 & $\mathrm{~N}$ & 3.779302 & 3.813785 & 6.401616 \\
\hline $\mathrm{N}$ & 2.859654 & 3.240034 & 5.612922 & $\mathrm{~N}$ & 2.676808 & 3.239593 & 5.851114 \\
\hline $\mathrm{Cu}$ & 3.845528 & 5.632902 & 7.127903 & $\mathrm{Cu}$ & 3.812945 & 5.691587 & 7.069866 \\
\hline $\mathrm{N}$ & 2.198073 & 5.589067 & 8.254971 & $\mathrm{~N}$ & 2.312762 & 5.581482 & 8.323620 \\
\hline $\mathrm{N}$ & 1.546706 & 6.696635 & 8.633298 & $\mathrm{~N}$ & 1.651323 & 6.713473 & 8.718619 \\
\hline $\mathrm{C}$ & 0.515127 & 6.355345 & 9.442977 & $\mathrm{C}$ & 0.683088 & 6.351075 & 9.588279 \\
\hline$C$ & 0.500832 & 4.964193 & 9.588451 & $C$ & 0.705819 & 4.962670 & 9.764598 \\
\hline C & 1.582018 & 4.521002 & 8.816488 & $C$ & 1.746457 & 4.518630 & 8.945395 \\
\hline $\mathrm{Cu}$ & 2.107552 & 8.441199 & 7.996053 & $\mathrm{Cu}$ & 2.163416 & 8.454585 & 7.993840 \\
\hline $\mathrm{O}$ & 2.763954 & 10.039499 & 7.178570 & 0 & 2.883973 & 10.106099 & 7.234594 \\
\hline $\mathrm{C}$ & 3.779631 & 9.872066 & 6.362318 & $C$ & 3.889475 & 9.938338 & 6.426274 \\
\hline C & 4.299843 & 8.442523 & 6.234497 & C & 4.401835 & 8.499467 & 6.266384 \\
\hline 0 & 3.576136 & 7.581328 & 7.120858 & 0 & 3.450178 & 7.573139 & 6.776153 \\
\hline O & 0.804917 & 9.421773 & 9.077753 & 0 & 0.841509 & 9.468202 & 9.078517 \\
\hline$C$ & -0.272797 & 9.759287 & 8.447457 & $C$ & -0.237974 & 9.692493 & 8.410832 \\
\hline$C$ & -1.283132 & 10.643303 & 9.182226 & $C$ & -1.359048 & 10.490394 & 9.079009 \\
\hline $\mathrm{Cl}$ & -0.836867 & 11.133424 & 10.879939 & $\mathrm{Cl}$ & -1.057565 & 10.998201 & 10.804118 \\
\hline $\mathrm{O}$ & 5.946522 & 5.641818 & 7.065552 & 0 & 5.881025 & 5.730354 & 6.853271 \\
\hline C & 6.837309 & 6.443022 & 7.895872 & $\mathrm{C}$ & 6.678946 & 6.221389 & 7.975841 \\
\hline $\mathrm{O}$ & 4.286182 & 10.808684 & 5.731260 & $\mathrm{O}$ & 4.459324 & 10.861135 & 5.815467 \\
\hline 0 & -0.584748 & 9.431312 & 7.285451 & 0 & -0.470457 & 9.310924 & 7.242473 \\
\hline $\mathrm{H}$ & 1.954696 & 3.516007 & 8.642194 & $\mathrm{H}$ & 2.120315 & 3.513397 & 8.776368 \\
\hline $\mathrm{H}$ & -0.197196 & 4.365021 & 10.164811 & $\mathrm{H}$ & 0.060157 & 4.362833 & 10.398341 \\
\hline $\mathrm{H}$ & -0.135402 & 7.116408 & 9.859660 & $\mathrm{H}$ & 0.043695 & 7.104530 & 10.037754 \\
\hline $\mathrm{H}$ & 5.626246 & 2.934781 & 7.153738 & $\mathrm{H}$ & 5.696782 & 3.057049 & 6.848009 \\
\hline $\mathrm{H}$ & 2.381246 & 1.369104 & 4.726936 & $\mathrm{H}$ & 2.123158 & 1.345906 & 5.066343 \\
\hline $\mathrm{H}$ & 6.339818 & 5.568265 & 6.174212 & $\mathrm{H}$ & 6.190663 & 6.172870 & 6.037704 \\
\hline $\mathrm{H}$ & 2.078171 & 3.819822 & 5.321207 & $\mathrm{H}$ & 1.831643 & 3.786194 & 5.708995 \\
\hline $\mathrm{H}$ & 4.776198 & 0.624617 & 5.906285 & $\mathrm{H}$ & 4.719231 & 0.704690 & 5.791305 \\
\hline $\mathrm{H}$ & 4.181781 & 8.125904 & 5.185499 & $\mathrm{H}$ & 4.607844 & 8.295843 & 5.204414 \\
\hline $\mathrm{H}$ & 5.371475 & 8.447816 & 6.484742 & $\mathrm{H}$ & 5.356255 & 8.462074 & 6.819485 \\
\hline $\mathrm{H}$ & 6.999044 & 7.435148 & 7.456452 & $\mathrm{H}$ & 6.478552 & 7.283108 & 8.166158 \\
\hline $\mathrm{H}$ & 7.796003 & 5.921654 & 8.002350 & $\mathrm{H}$ & 7.739796 & 6.070898 & 7.750669 \\
\hline $\mathrm{H}$ & 6.354418 & 6.541224 & 8.871325 & $\mathrm{H}$ & 6.390790 & 5.624015 & 8.845074 \\
\hline $\mathrm{H}$ & -2.238659 & 10.113157 & 9.246677 & $\mathrm{H}$ & -2.273277 & 9.888483 & 9.083266 \\
\hline $\mathrm{H}$ & -1.421349 & 11.568050 & 8.612921 & $\mathrm{H}$ & -1.535049 & 11.406482 & 8.506235 \\
\hline
\end{tabular}


(a)

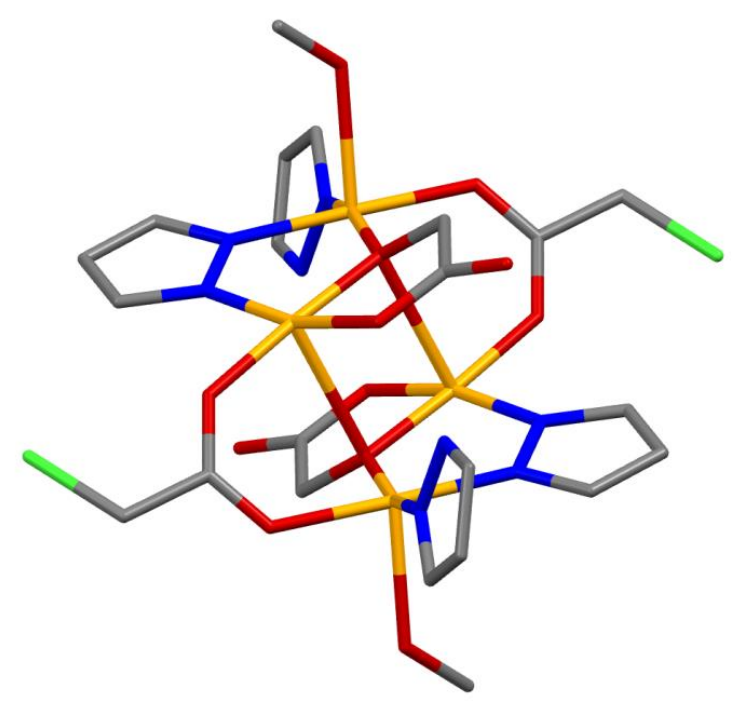

(b)

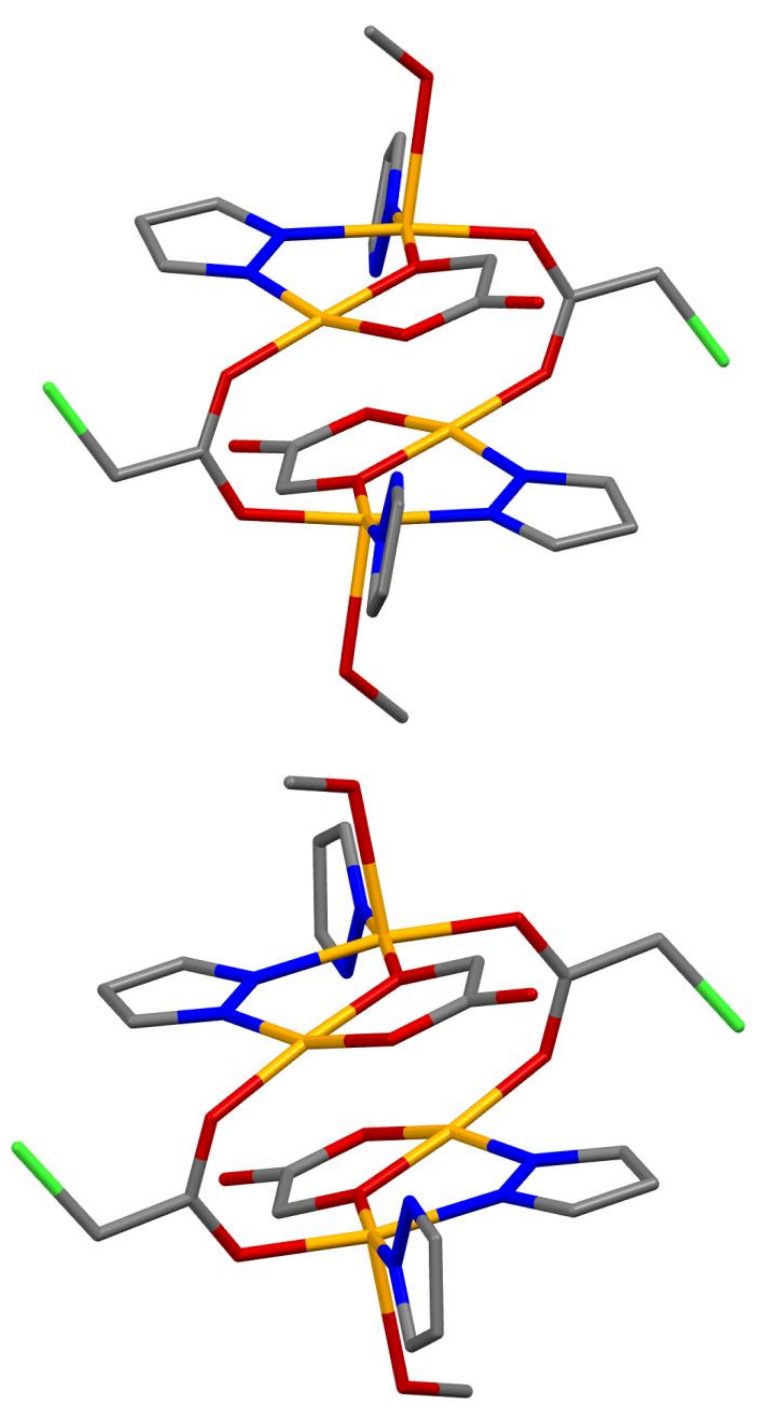

Figure $\mathbf{S 8 ~ - ~ L i c o r i c e ~ r e p r e s e n t a t i o n ~ o f ~} \mathbf{3 t}$ with the inclusion of solvent effects; (a) experimental coordinates, (b) coordinates optimized by assuming an antiferromagnetic coupling $(S=0)$, (c) coordinates optimized by assuming $\mathrm{S}=\mathrm{O}$ and adding a dispersion correction. $\mathrm{H}$ atoms are not reported for the sake of clarity. 
Table S3 - Optimized Cartesian coordinates of $\mathbf{3 t}$ with the inclusion of solvent effects and by assuming an antiferromagnetic coupling $(S=0)$, with/without dispersion correction.

\begin{tabular}{|c|c|c|c|c|c|c|c|}
\hline \multicolumn{4}{|c|}{ Without dispersion correction } & \multicolumn{4}{|c|}{ With dispersion correction } \\
\hline C & 0.023259 & -3.124040 & 2.885068 & $C$ & -0.315984 & -3.016389 & 2.465192 \\
\hline $\mathrm{H}$ & 0.402131 & -4.125468 & 2.709084 & $\mathrm{H}$ & -0.085780 & -4.023773 & 2.170011 \\
\hline C & -0.877542 & -2.652571 & 3.848670 & $C$ & -1.125459 & -2.550586 & 3.499066 \\
\hline $\mathrm{H}$ & -1.375962 & -3.221737 & 4.627533 & $\mathrm{H}$ & -1.678871 & -3.133929 & 4.215145 \\
\hline$C$ & -0.992622 & -1.285110 & 3.578411 & $C$ & -1.059998 & -1.163111 & 3.399537 \\
\hline $\mathrm{H}$ & -1.577317 & -0.513358 & 4.069555 & $\mathrm{H}$ & -1.518363 & -0.394651 & 3.997010 \\
\hline C & 2.299244 & -5.134102 & 0.499728 & $C$ & 2.200143 & -4.944847 & 0.628004 \\
\hline $\mathrm{H}$ & 3.218505 & -4.972797 & 1.053386 & $\mathrm{H}$ & 3.115706 & -4.717390 & 1.144667 \\
\hline C & 1.751463 & -6.314758 & -0.032607 & $C$ & 1.589918 & -6.179842 & 0.374462 \\
\hline $\mathrm{H}$ & 2.164026 & -7.316671 & 0.016107 & $\mathrm{H}$ & 1.948076 & -7.157328 & 0.645943 \\
\hline C & 0.560998 & -5.923447 & -0.639844 & $C$ & 0.419264 & -5.874736 & -0.298381 \\
\hline $\mathrm{H}$ & -0.192507 & -6.486944 & -1.179533 & $\mathrm{H}$ & -0.363758 & -6.499462 & -0.690065 \\
\hline C & 2.608813 & 0.723158 & 0.191756 & $C$ & 2.534746 & 0.848653 & 0.022812 \\
\hline $\mathrm{H}$ & 2.702191 & 0.579857 & -0.894395 & $\mathrm{H}$ & 2.499160 & 0.785950 & -1.063033 \\
\hline $\mathrm{H}$ & 3.596405 & 0.554913 & 0.650140 & $\mathrm{H}$ & 3.542774 & 0.593925 & 0.356362 \\
\hline C & 2.217647 & 2.181071 & 0.427165 & $C$ & 2.260289 & 2.299745 & 0.400590 \\
\hline C & -2.444562 & 1.973932 & 2.268134 & $C$ & -2.382308 & 1.893987 & 2.374429 \\
\hline C & -3.532144 & 2.379579 & 3.265457 & $C$ & -3.460053 & 2.131834 & 3.419579 \\
\hline $\mathrm{H}$ & -4.434603 & 1.789331 & 3.079985 & $\mathrm{H}$ & -4.287063 & 1.449661 & 3.253042 \\
\hline $\mathrm{H}$ & -3.765520 & 3.440534 & 3.124577 & $\mathrm{H}$ & -3.816219 & 3.155186 & 3.334633 \\
\hline C & 3.886615 & -2.114779 & 3.193190 & $C$ & 3.158337 & -1.472517 & 3.121084 \\
\hline $\mathrm{H}$ & 4.842633 & -2.435509 & 3.629507 & $\mathrm{H}$ & 3.971055 & -1.623825 & 3.829470 \\
\hline $\mathrm{H}$ & 3.779676 & -1.025555 & 3.297492 & $\mathrm{H}$ & 3.063444 & -0.415145 & 2.881844 \\
\hline $\mathrm{H}$ & 3.064532 & -2.606036 & 3.723790 & $\mathrm{H}$ & 2.227971 & -1.822033 & 3.555858 \\
\hline$N$ & 0.415206 & -2.091626 & 2.102293 & $\mathrm{~N}$ & 0.184838 & -1.962937 & 1.798622 \\
\hline $\mathrm{N}$ & -0.199826 & -0.979290 & 2.520209 & $\mathrm{~N}$ & -0.256648 & -0.850144 & 2.367034 \\
\hline $\mathrm{N}$ & 1.491546 & -4.090237 & 0.236376 & $\mathrm{~N}$ & 1.452318 & -3.959087 & 0.133765 \\
\hline$N$ & 0.438880 & -4.589950 & -0.457127 & $\mathrm{~N}$ & 0.373148 & -4.540620 & -0.424120 \\
\hline $\mathrm{H}$ & -0.295809 & -3.922825 & -0.771890 & $\mathrm{H}$ & -0.356744 & -3.933712 & -0.825627 \\
\hline 0 & 1.625373 & -0.150301 & 0.751855 & $\mathrm{O}$ & 1.574582 & -0.013182 & 0.618401 \\
\hline 0 & 1.093507 & 2.377073 & 1.085926 & $\mathrm{O}$ & 1.248085 & 2.493377 & 1.201759 \\
\hline 0 & 2.925645 & 3.105289 & 0.014958 & $\mathrm{O}$ & 2.956409 & 3.204547 & -0.032961 \\
\hline 0 & -1.277938 & 1.714264 & 2.689006 & $\mathrm{O}$ & -1.180891 & 1.845163 & 2.729856 \\
\hline 0 & -2.868315 & 1.964303 & 1.066703 & $\mathrm{O}$ & -2.841001 & 1.780729 & 1.205865 \\
\hline 0 & 3.789825 & -2.525439 & 1.807866 & $\mathrm{O}$ & 3.374198 & -2.251395 & 1.928072 \\
\hline $\mathrm{Cl}$ & -3.086873 & 2.156378 & 5.007783 & $\mathrm{Cl}$ & -2.897659 & 1.899393 & 5.120065 \\
\hline $\mathrm{Cu}$ & 0.216561 & 0.760974 & 1.726061 & $\mathrm{Cu}$ & 0.278532 & 0.898774 & 1.709204 \\
\hline $\mathrm{Cu}$ & 1.706021 & -2.098282 & 0.563215 & $\mathrm{Cu}$ & 1.623898 & -1.948927 & 0.380559 \\
\hline $\mathrm{H}$ & 4.541577 & -2.127155 & 1.329611 & $\mathrm{H}$ & 4.220230 & -1.981616 & 1.540335 \\
\hline C & -0.023259 & 3.124040 & -2.885068 & $\mathrm{C}$ & 0.315984 & 3.016389 & -2.465192 \\
\hline $\mathrm{H}$ & -0.402131 & 4.125468 & -2.709084 & $\mathrm{H}$ & 0.085780 & 4.023773 & -2.170011 \\
\hline$C$ & 0.877542 & 2.652571 & -3.848670 & $\mathrm{C}$ & 1.125459 & 2.550586 & -3.499066 \\
\hline $\mathrm{H}$ & 1.375962 & 3.221737 & -4.627533 & $\mathrm{H}$ & 1.678871 & 3.133929 & -4.215145 \\
\hline C & 0.992622 & 1.285110 & -3.578411 & $\mathrm{C}$ & 1.059998 & 1.163111 & -3.399537 \\
\hline $\mathrm{H}$ & 1.577317 & 0.513358 & -4.069555 & $\mathrm{H}$ & 1.518363 & 0.394651 & -3.997010 \\
\hline C & -2.299244 & 5.134102 & -0.499728 & $\mathrm{C}$ & -2.200143 & 4.944847 & -0.628004 \\
\hline $\mathrm{H}$ & -3.218505 & 4.972797 & -1.053386 & $\mathrm{H}$ & -3.115706 & 4.717390 & -1.144667 \\
\hline C & -1.751463 & 6.314758 & 0.032607 & $\mathrm{C}$ & -1.589918 & 6.179842 & -0.374462 \\
\hline $\mathrm{H}$ & -2.164026 & 7.316671 & -0.016107 & $\mathrm{H}$ & -1.948076 & 7.157328 & -0.645943 \\
\hline C & -0.560998 & 5.923447 & 0.639844 & $\mathrm{C}$ & -0.419264 & 5.874736 & 0.298381 \\
\hline $\mathrm{H}$ & 0.192507 & 6.486944 & 1.179533 & $\mathrm{H}$ & 0.363758 & 6.499462 & 0.690065 \\
\hline C & -2.608813 & -0.723158 & -0.191756 & $\mathrm{C}$ & -2.534746 & -0.848653 & -0.022812 \\
\hline $\mathrm{H}$ & -2.702191 & -0.579857 & 0.894395 & $\mathrm{H}$ & -2.499160 & -0.785950 & 1.063033 \\
\hline $\mathrm{H}$ & -3.596405 & -0.554913 & -0.650140 & $\mathrm{H}$ & -3.542774 & -0.593925 & -0.356362 \\
\hline C & -2.217647 & -2.181071 & -0.427165 & $\mathrm{C}$ & -2.260289 & -2.299745 & -0.400590 \\
\hline C & 2.444562 & -1.973932 & -2.268134 & $\mathrm{C}$ & 2.382308 & -1.893987 & -2.374429 \\
\hline C & 3.532144 & -2.379579 & -3.265457 & C & 3.460053 & -2.131834 & -3.419579 \\
\hline $\mathrm{H}$ & 4.434603 & -1.789331 & -3.079985 & $\mathrm{H}$ & 4.287063 & -1.449661 & -3.253042 \\
\hline $\mathrm{H}$ & 3.765520 & -3.440534 & -3.124577 & $\mathrm{H}$ & 3.816219 & -3.155186 & -3.334633 \\
\hline C & -3.886615 & 2.114779 & -3.193190 & $\mathrm{C}$ & -3.158337 & 1.472517 & -3.121084 \\
\hline $\mathrm{H}$ & -4.842633 & 2.435509 & -3.629507 & $\mathrm{H}$ & -3.971055 & 1.623825 & -3.829470 \\
\hline $\mathrm{H}$ & -3.779676 & 1.025555 & -3.297492 & $\mathrm{H}$ & -3.063444 & 0.415145 & -2.881844 \\
\hline $\mathrm{H}$ & -3.064532 & 2.606036 & -3.723790 & $\mathrm{H}$ & -2.227971 & 1.822033 & -3.555858 \\
\hline$N$ & -0.415206 & 2.091626 & -2.102293 & $\mathrm{~N}$ & -0.184838 & 1.962937 & -1.798622 \\
\hline$N$ & 0.199826 & 0.979290 & -2.520209 & $N$ & 0.256648 & 0.850144 & -2.367034 \\
\hline $\mathrm{N}$ & -1.491546 & 4.090237 & -0.236376 & $\mathrm{~N}$ & -1.452318 & 3.959087 & -0.133765 \\
\hline $\mathrm{N}$ & -0.438880 & 4.589950 & 0.457127 & $\mathrm{~N}$ & -0.373148 & 4.540620 & 0.424120 \\
\hline
\end{tabular}




\begin{tabular}{|llll|llll|}
\hline $\mathrm{H}$ & 0.295809 & 3.922825 & 0.771890 & $\mathrm{H}$ & 0.356744 & 3.933712 & 0.825627 \\
$\mathrm{O}$ & -1.625373 & 0.150301 & -0.751855 & $\mathrm{O}$ & -1.574582 & 0.013182 & -0.618401 \\
$\mathrm{O}$ & -1.093507 & -2.377073 & -1.085926 & $\mathrm{O}$ & -1.248085 & -2.493377 & -1.201759 \\
$\mathrm{O}$ & -2.925645 & -3.105289 & -0.014958 & $\mathrm{O}$ & -2.956409 & -3.204547 & 0.032961 \\
$\mathrm{O}$ & 1.277938 & -1.714264 & -2.689006 & $\mathrm{O}$ & 1.180891 & -1.845163 & -2.729856 \\
$\mathrm{O}$ & 2.868315 & -1.964303 & -1.066703 & $\mathrm{O}$ & 2.841001 & -1.780729 & -1.205865 \\
$\mathrm{O}$ & -3.789825 & 2.525439 & -1.807866 & $\mathrm{O}$ & -3.374198 & 2.251395 & -1.928072 \\
$\mathrm{Cl}$ & 3.086873 & -2.156378 & -5.007783 & $\mathrm{Cl}$ & 2.897659 & -1.899393 & -5.120065 \\
$\mathrm{Cu}$ & -0.216561 & -0.760974 & -1.726061 & $\mathrm{Cu}$ & -0.278532 & -0.898774 & -1.709204 \\
$\mathrm{Cu}$ & -1.706021 & 2.098282 & -0.563215 & $\mathrm{Cu}$ & -1.623898 & 1.948927 & -0.380559 \\
$\mathrm{H}$ & -4.541577 & 2.127155 & -1.329611 & $\mathrm{H}$ & -4.220230 & 1.981616 & -1.540335 \\
\hline
\end{tabular}

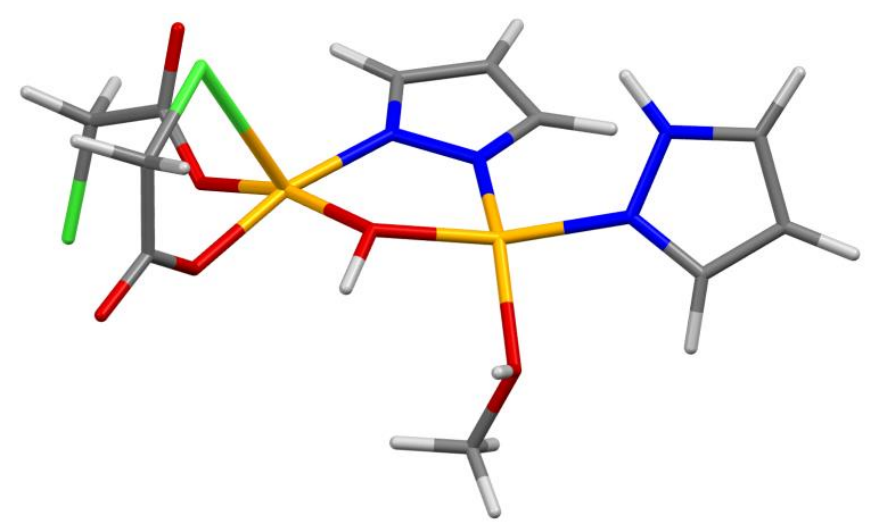

Figure $\mathbf{S 9}$ - Optimized structure of the reaction intermediate ${ }^{1} \mathbf{3} \mathbf{t}^{\mathrm{i}}$ with the inclusion of solvent (methanol) effects and by assuming a ferromagnetic coupling $(S=1)$. 
Table S4 - Optimized Cartesian coordinates of the stable intermediate $\mathbf{3 t}$ ' for mechanism in Scheme 2 with the inclusion of solvent effects and by assuming either an antiferromagnetic $(S=0)$ or a ferromagnetic $(S=$ 1) coupling.

\begin{tabular}{|c|c|c|c|c|c|c|c|}
\hline$S=0$ & & & & $S=1$ & & & \\
\hline $\mathrm{N}$ & 3.159708 & 3.382321 & 5.581030 & $\mathrm{~N}$ & 3.288384 & 3.123545 & 5.781265 \\
\hline $\mathrm{N}$ & 4.101583 & 3.889698 & 6.419481 & $\mathrm{~N}$ & 4.140240 & 3.796439 & 6.598330 \\
\hline$C$ & 5.016741 & 2.910278 & 6.566598 & C & 5.015472 & 2.876674 & 7.052789 \\
\hline C & 4.655223 & 1.781650 & 5.814061 & C & 4.722516 & 1.616481 & 6.510872 \\
\hline C & 3.458203 & 2.123130 & 5.193720 & $\mathrm{C}$ & 3.612694 & 1.815623 & 5.696100 \\
\hline $\mathrm{Cu}$ & 4.017434 & 5.721802 & 7.205448 & $\mathrm{Cu}$ & 3.922677 & 5.710318 & 7.049160 \\
\hline 0 & 6.103493 & 5.802573 & 7.407042 & O & 5.996916 & 5.853555 & 7.334436 \\
\hline C & 6.637877 & 6.223798 & 8.703052 & C & 6.483189 & 6.550483 & 8.525784 \\
\hline $\mathrm{N}$ & 2.127641 & 5.703653 & 7.905438 & $\mathrm{~N}$ & 2.107559 & 5.627406 & 7.779639 \\
\hline C & 1.334926 & 4.649622 & 8.222295 & $C$ & 1.344451 & 4.576362 & 8.165933 \\
\hline C & 0.103295 & 5.124397 & 8.689253 & $C$ & 0.102182 & 5.043930 & 8.599981 \\
\hline C & 0.221792 & 6.517478 & 8.626648 & $\mathrm{C}$ & 0.172023 & 6.433688 & 8.444359 \\
\hline $\mathrm{N}$ & 1.448229 & 6.831082 & 8.145450 & $\mathrm{~N}$ & 1.379513 & 6.777672 & 7.945702 \\
\hline $\mathrm{Cu}$ & 2.257162 & 8.600715 & 7.837722 & $\mathrm{Cu}$ & 2.228981 & 8.534901 & 7.659931 \\
\hline 0 & 3.237982 & 10.247157 & 7.478970 & 0 & 3.406727 & 10.135360 & 7.539531 \\
\hline C & 3.502973 & 10.792952 & 6.335065 & $\mathrm{C}$ & 3.648214 & 10.908176 & 6.537768 \\
\hline C & 2.889128 & 10.304991 & 5.020401 & $C$ & 2.861424 & 10.820718 & 5.225997 \\
\hline $\mathrm{Cl}$ & 1.500157 & 9.135126 & 5.157812 & $\mathrm{Cl}$ & 1.392284 & 9.739740 & 5.231152 \\
\hline 0 & 3.826908 & 7.621933 & 7.353988 & 0 & 3.690207 & 7.573133 & 6.675313 \\
\hline 0 & 1.058676 & 9.579444 & 9.045542 & 0 & 1.140019 & 9.535704 & 8.984976 \\
\hline C & -0.119051 & 10.027156 & 8.781489 & $\mathrm{C}$ & -0.093674 & 9.866074 & 8.843312 \\
\hline C & -0.753649 & 10.979502 & 9.806099 & C & -0.718422 & 10.766155 & 9.918897 \\
\hline $\mathrm{Cl}$ & 0.233144 & 11.341069 & 11.298688 & $\mathrm{Cl}$ & 0.376397 & 11.273714 & 11.289748 \\
\hline $\mathrm{O}$ & -0.825175 & 9.776547 & 7.785383 & 0 & -0.872708 & 9.536645 & 7.924091 \\
\hline 0 & 4.291758 & 11.753570 & 6.219275 & 0 & 4.546407 & 11.779780 & 6.537962 \\
\hline $\mathrm{H}$ & 1.698169 & 3.632397 & 8.111573 & $\mathrm{H}$ & 1.729430 & 3.562580 & 8.116861 \\
\hline $\mathrm{H}$ & -0.752723 & 4.545331 & 9.022296 & $\mathrm{H}$ & -0.732322 & 4.458256 & 8.972257 \\
\hline $\mathrm{H}$ & -0.490589 & 7.297502 & 8.871423 & $\mathrm{H}$ & -0.569195 & 7.200984 & 8.644316 \\
\hline $\mathrm{H}$ & 5.882817 & 3.073402 & 7.199280 & $\mathrm{H}$ & 5.803540 & 3.172571 & 7.736648 \\
\hline $\mathrm{H}$ & 2.810220 & 1.577423 & 4.516909 & $\mathrm{H}$ & 3.042930 & 1.138844 & 5.068995 \\
\hline $\mathrm{H}$ & 6.539170 & 6.336491 & 6.713179 & $\mathrm{H}$ & 6.458981 & 6.223034 & 6.554921 \\
\hline H & 2.359328 & 3.948465 & 5.313184 & $\mathrm{H}$ & 2.540201 & 3.618518 & 5.303401 \\
\hline $\mathrm{H}$ & 5.191372 & 0.843032 & 5.730763 & $\mathrm{H}$ & 5.248776 & 0.684645 & 6.683646 \\
\hline $\mathrm{H}$ & 2.514625 & 11.169609 & 4.466149 & $\mathrm{H}$ & 2.507625 & 11.819073 & 4.954026 \\
\hline $\mathrm{H}$ & 3.664003 & 9.806104 & 4.427926 & $\mathrm{H}$ & 3.522098 & 10.444183 & 4.438386 \\
\hline $\mathrm{H}$ & 7.725650 & 6.091510 & 8.700291 & $\mathrm{H}$ & 7.566270 & 6.406015 & 8.603349 \\
\hline $\mathrm{H}$ & 6.184782 & 5.568056 & 9.451956 & $\mathrm{H}$ & 5.981661 & 6.090115 & 9.381377 \\
\hline $\mathrm{H}$ & 6.377892 & 7.267490 & 8.913349 & $\mathrm{H}$ & 6.247632 & 7.620615 & 8.483252 \\
\hline $\mathrm{H}$ & 4.590257 & 8.206104 & 7.162148 & $\mathrm{H}$ & 4.491253 & 8.103370 & 6.861933 \\
\hline $\mathrm{H}$ & -1.701077 & 10.554896 & 10.152719 & $\mathrm{H}$ & -1.565875 & 10.244168 & 10.375021 \\
\hline $\mathrm{H}$ & -0.945448 & 11.941462 & 9.319828 & $\mathrm{H}$ & -1.074588 & 11.687720 & 9.447669 \\
\hline
\end{tabular}

